\title{
Supporting Information Life Span of Slippery Lubricant Infused Surfaces
}

Muhammad Jahidul Hoque ${ }^{1}$, Soumyadip Sett ${ }^{1,2}$, Xiao Yan ${ }^{1}$, Derrick Liu ${ }^{1}$, Kazi Fazle Rabbi ${ }^{1}$, Haoyun Qiu ${ }^{1}$, Mansoor Qureshi ${ }^{3}$, George Barac ${ }^{4}$, Leslie Bolton ${ }^{5}$, and Nenad Miljkovic ${ }^{1,6,7,8 *}$

${ }^{1}$ Department of Mechanical Science and Engineering, University of Illinois, Urbana, IL, USA ${ }^{2}$ Current Address: Department of Mechanical Engineering, IIT Gandhinagar, Ahmedabad,India ${ }^{3}$ Department of Chemistry, University of Illinois, Urbana, IL, USA

${ }^{4} \mathrm{BP}$ International Limited, $150 \mathrm{~W}$ Warrenville Road, Naperville, Illinois 60563, USA ${ }^{5}$ BP plc, Chertsey Road, Sunbury-on-Thames, Middlesex TW16 7LN, UK ${ }^{6}$ Materials Research Laboratory, University of Illinois, Urbana, IL, USA

${ }^{7}$ Department of Electrical and Computer Engineering, University of Illinois, Urbana, IL, USA

${ }^{8}$ International Institute for Carbon Neutral Energy Research (WPI-I2CNER), Kyushu University, 744 Motooka, Nishi-ku, Fukuoka 819-0395, Japan

(*Corresponding Author’s E-mail: nmiljkov@illinois.edu) 


\section{S1.Details of Sample Fabrication}

Sample Preparation. The copper tubes used in these experiments were systematically cleaned prior to testing. Firstly, un-capped ttubes were cleaned by subsequently submersing them in acetone, ethanol, isopropyl alcohol (IPA) and de-ionized (DI) water for about 15 minutes each at room temperature. After rinsing the tubes in DI water and drying in a clean nitrogen stream, the tubes were dipped into a 2.0 M hydrochloric acid solution for 2 minutes to remove the native oxide film on the surface. Finally, the tubes were rinsed with DI water and dried with a clean nitrogen stream. Then all cleaned tubes were interfaced with a female 3/8" stainless steel Swagelok tube fitting on each end and capped with a 3/8" stainless steel Swagelok nut. Capping of the tubes was done before $\mathrm{CuO}$ fabrication to ensure no oxidation or functionalization occurred on the internal surface.

CuO Fabrication. Balde like copper oxide $(\mathrm{CuO})$ micro/nano-structure were formed on the cleaned capped copper samples by immersing the tubes in a hot $\left(90 \pm 5^{\circ} \mathrm{C}\right)$ alkaline solution composed of $\mathrm{NaClO}_{2}, \mathrm{NaOH}, \mathrm{Na}_{3} \mathrm{PO}_{4} \cdot 12 \mathrm{H}_{2} \mathrm{O}$, and DI water (3.75:5:10:100 wt\%). ${ }^{1-3}$ The oxidation process led to the formation of a thin $(\approx 300 \mathrm{~nm}) \mathrm{Cu}_{2} \mathrm{O}$ layer that reoxidized to form sharp, knife-like $\mathrm{CuO}$ structures $(h \approx 1 \mu \mathrm{m}$, solid fraction $\varphi \approx 0.023$, and roughness factor $r \approx 10){ }^{4}$

Silanization. The nanostructured $\mathrm{CuO}$ tubes were functionalized using atmospheric pressure chemical vapor deposition (CVD) of a fluorinated silane (heptadecafluorodecyltrimethoxy-silane, abbreviated as HTMS, Gelest, CAS \#83048-65-1). ${ }^{5}$ The sample tubes were placed vertically inside a tall glass beaker. Along with the tube samples, $10 \mathrm{~mL}$ of a HTMS-toluene solution (5\% v/v) was placed in a small glass vial inside the beaker. A lid was placed on top to seal the container, followed by heating in an atmospheric pressure oven (Thermo Scientific, Lindberg Blue M) at $80 \pm 5^{\circ} \mathrm{C}$ for 3 hours to allow conformal HTMS SAM deposition. 
Slippery Lubricant-Infused Porous Surfaces (SLIPS).The functionalized nanostructured CuO tube samples were dip coated to infuse the surface with the lubricant of choice. The tube samples were immersed in the lubricant for 10 mins and then removed and left in a vertical position for 24 hours in ambient conditions to allow gravitational drainage of excess lubricant. The SLIPS samples were then dried in a clean nitrogen stream and tested in the chamber.

Silicon Based SHP and SLIPS. Silicon wafer was cleaned by subsequently submersing them in acetone, ethanol, isopropyl alcohol (IPA) and de-ionized (DI) water for about 15 minutes each at room temperature. Thin aluminum film layer $(50 \mathrm{~nm})$ is deposited on top of silicon wafer by sputtering process. The sputtering system (AJA Orion-8 Magnetron) is operated with RF power 200W. The chamber vacuum is maintained with 3 mTorr and Argon gas is introduced to generate plasma with mass flow rate of $30 \mathrm{sccm}$. The thickness of deposited aluminum layer is controlled by sputtering time. To create aluminum boehmite nanostructures on the surfaces, sputter-coated aluminum samples are immersed in hot $\left(90^{\circ} \mathrm{C}\right)$ DI water for 10 minutes followed by rising with DI water and drying with clean nitrogen flow. Then, the surfaces are functionalized with Heptadecafluorodecyltrimethoxy-silane (CAS No. 83048-65-1) through well known CVD process. ${ }^{5}$ Then the surface is immersed in the lubricant for 10 mins and then removed and left in a vertical position for 24 hours in ambient conditions to allow gravitational drainage of excess lubricant. The sample is dried in a clean nitrogen stream.

Aluminum Based SHP and SLIPS. Firstly, commerically available Aluminum coupon sample $(15 \mathrm{~mm} \times 15 \mathrm{~mm})$ cleaned by subsequently submersing them in acetone, ethanol, isopropyl alcohol (IPA) and de-ionized (DI) water for about 15 minutes each at room temperature. Then to create aluminum boehmite nanostructures on the surfaces, cleaned aluminum samples are immersed in hot $\left(90^{\circ} \mathrm{C}\right) \mathrm{DI}$ water for 60 minutes followed by rising with DI water and drying with clean nitrogen 
flow. Then, the surfaces are functionalized with Heptadecafluorodecyltrimethoxy-silane (CAS No. 83048-65-1) through well known CVD process. ${ }^{5}$ Finally, the surface is immersed in the lubricant for 10 mins and then removed and left in a vertical position for 24 hours in ambient conditions to allow gravitational drainage of excess lubricant. Then the sample is dried in a clean nitrogen stream.

To study the surface morphology using SEM and to measure contact angles using a microgoniometer, additional flat small samples $(15 \mathrm{~mm} \times 15 \mathrm{~mm})$ were fabricated using the same procedures described above. 


\section{S2.Properties of Infused Oils}

Table S1. Physical Properties of Different Infused Oil at Room Temperature $\left(\sim 20^{\circ} \mathrm{C}\right)$.

\begin{tabular}{|c|c|c|c|c|c|c|c|}
\hline \multirow{2}{*}{$\begin{array}{l}\text { Infused } \\
\text { Oil }\end{array}$} & \multirow{2}{*}{ Oil Type } & \multirow{2}{*}{$\begin{array}{l}\text { Density } \\
{\left[\mathrm{Kg} / \mathrm{m}^{3}\right]}\end{array}$} & \multirow{2}{*}{\begin{tabular}{c}
\multicolumn{1}{c}{ Oil } \\
Dynamic \\
Viscosity \\
{$[\mathrm{mPa} \cdot \mathrm{s}]$}
\end{tabular}} & \multirow{2}{*}{$\begin{array}{l}\text { Surface } \\
\text { Tension } \\
{[\mathrm{mN} / \mathrm{m}]}\end{array}$} & \multirow{2}{*}{$\begin{array}{c}\text { Vapor Pressure at } \\
20^{\circ} \mathrm{C}[\mathrm{kPa}]\end{array}$} & \multicolumn{2}{|c|}{ Spreading Coefficient } \\
\hline & & & & & & Water & Ethanol \\
\hline $\begin{array}{c}\text { Carnation } \\
\text { Oil } \\
\end{array}$ & Mineral & 810 & 9.7 & 28 & $10^{-2}$ & -6.6 & -10.11 \\
\hline GPL101 & \multirow{4}{*}{ Fluorinated } & 1890 & 14.7 & 18.8 & N/A & 2.9 & 0.0 \\
\hline $\begin{array}{c}\text { Fomblin } \\
\text { Y25/6 }\end{array}$ & & 1900 & 524 & 20 & $6 \times 10^{-8}$ & 1.76 & -1.22 \\
\hline $\begin{array}{c}\text { Krytox } \\
1525 \\
\end{array}$ & & 1900 & 496 & 19 & $1.3 \times 10^{-8}$ & 2.70 & -0.20 \\
\hline $\begin{array}{c}\text { Krytox } \\
16256\end{array}$ & & 1920 & 5216 & 19 & $4 \times 10^{-15}$ & 2.70 & -0.20 \\
\hline
\end{tabular}




\section{S3. Effect of Exposure Temperature on Oil Viscosity}

To characterize the effect of temperature exposure on oil viscosity (i.e. degradation or change of oil properties due to harsh condition exposure), we have conducted experiments by exposing the oils (carnation and Krytox 16256 oil) to $-50^{\circ} \mathrm{C}$ and $200^{\circ} \mathrm{C}$ for 24 hours. After exposure, we allow the oils to come back to room temperature $\left(20^{\circ} \mathrm{C}\right)$, and we experimentally measured the viscosity using a rheometer (ARES-G2). Irrespective of the exposure temperature, the viscosity was similar to the measured fresh oil samples (see Table S2).

Table S2. Experimentally measured viscosity of two oils after exposure to different temperatures and allowing them to settle back to room temperature.

\begin{tabular}{|c|c|c|}
\hline \multirow{2}{*}{$\begin{array}{c}\text { Exposure } \\
\text { Temperature }\left[{ }^{\circ} \mathrm{C}\right]\end{array}$} & \multicolumn{2}{|c|}{ Measured Viscosity after Exposure [mPa·s] } \\
\cline { 2 - 3 } & Carnation Oil & Krytox 16256 \\
\hline-50 & 9.689 & 5216.008 \\
\hline 20 & 9.693 & 5215.987 \\
\hline 200 & 9.702 & 5215.991 \\
\hline
\end{tabular}




\section{S4. Scaling Analysis of Potential Oil Depletion Mechanisms}

Cloak layer volume. The volume of oil depletion due to the oil cloak layer can be calculated by comparing the size of a cloaked condensate droplet to the non-cloaked droplet. The volume of a condensate droplet $\left(V_{\text {droplet }}\right)$ can be expressed as: ${ }^{6}$

$$
V_{\text {droplet }}=\frac{\pi}{3}\left[(1-\cos \theta)^{2}(2+\cos \theta) R^{3}\right]
$$

where $\theta$ and $R$ are the apparent advancing contact angle and droplet radius, respectively. Assuming a cloak layer on the outside of the droplet with a uniform layer thickness $(t)$, the total volume of the cloaked droplet can be written as:

$$
V_{\text {cloaked droplet }}=\frac{\pi}{3}\left[(1-\cos \theta)^{2}(2+\cos \theta)(R+t)^{3}\right]
$$

Now, volume of the cloak layer volume ( $\left.V_{\text {cloak layer }}\right)$ can be obtained by subtracting Eq. (S2) from (S1), and can be expressed as:

$$
V_{\text {cloak layer }}=\frac{\pi}{3}\left[(1-\cos \theta)^{2}(2+\cos \theta)\right]\left[(R+t)^{3}-R^{3}\right]
$$

Assuming a cloaked condensate with a contact angle of $120^{\circ}$ and droplet diameter of $2 \mathrm{~mm}$ having a $10 \mathrm{~nm}$ thick cloak layer, $V_{\text {cloak layer }}=1.06 \times 10^{-13} \mathrm{~m}^{3}$

Oil ridge volume. The volume of the wetting ridge around a droplet can be expressed as a function of the capillary number by: ${ }^{7}$

$$
V_{\text {ridge }}=\frac{R_{\mathrm{b}} h_{\mathrm{i}}^{2}}{\mu_{1} C a^{\frac{4}{3}}}
$$

where $R_{\mathrm{b}}$ is the base radius of the droplet, $h_{\mathrm{i}}$ is the initial lubricant film thickness, $\mu_{1} \sim 0.5$, is the limiting size of the wetting ridge for a given set of experimental conditions, ${ }^{7}$ and $\mathrm{Ca}=\eta U / \gamma$ is 
the capillary number; here $\eta$ is the oil viscosity, $\gamma$ is the surface tension of the oil, and $U$ is the droplet shedding velocity.

Assuming a SLIPS infused with K-16256 $(\eta=5216 \mathrm{mPa} \cdot \mathrm{s} ; \gamma=19 \mathrm{mN} / \mathrm{m})$ having an initial oil layer thickness, $h_{\mathrm{i}}=2 \mu \mathrm{m}$ and a condensate droplet diameter $2 \mathrm{~mm}$, which is shedding at a velocity of $2 \mathrm{~mm} / \mathrm{s}$, Eq. (S4) gives $V_{\text {ridge }}=1.78 \times 10^{-14} \mathrm{~m}^{3}<V_{\text {cloak layer }}=1.06 \times 10^{-13} \mathrm{~m}^{3}$. If the cloak layer and oil ridge volume are the only source of oil depletion from the SLIPS, for a high viscosity SLIPS (K-16256), the contribution of the cloak layer to the total oil depletion is $\sim 6 \mathrm{X}$ higher than the contribution from the oil ridge. This scenario will change if the oil viscosity is lowered just by an order of magnitude. For a SLIPS with moderate viscosity oil, K-1525 ( $\eta=496$ $\mathrm{mPa} . \mathrm{s} ; \gamma=19 \mathrm{mN} / \mathrm{m})$, assuming the same cloak layer thickness $(10 \mathrm{~nm})$, identical droplet diameter (2 $\mathrm{mm})$ and the same droplet shedding velocity $(2 \mathrm{~mm} / \mathrm{s})$, the oil ridge volume becomes comparable to cloak layer volume. $V_{\text {ridge }}=4.09 \times 10^{-13} \mathrm{~m}^{3} \sim V_{\text {cloak layer }}$.

Lifespan Prediction. By combining the estimated volume of oil drainage per droplet with the experimentally observed droplet count, we can predict the idealized lifespan of a SLIPS surface. In our condensation experiments in pure steam conditions, we observed that the number of droplet shedding events from a SLIPS infused with K-16256 was approximately 30 droplets/min. In pure ethanol condensation, this number was $\sim 60$ droplets/min. For both cases, the vapor pressure was maintained at $\sim 6 \mathrm{kPa}$ in an environmental chamber and experiments performed on a SLIPS tube sample having 3/8" $(0.0095 \mathrm{~m})$ external diameter with 3" $(0.0762 \mathrm{~m})$ length. In our simplified scaling analysis, we showed that for K-16256 infused SLIPS, the volume of oil drainage due to the cloak layer and oil-ridge induced droplet shedding are, $V_{\text {cloaklayer }}=1.06 \times 10^{-13} \mathrm{~m}^{3}$ and $V_{\text {ridge }}=1.78 \times 10^{-14} \mathrm{~m}^{3}$, respectively 
Due to the negative spreading coefficient of K-16256 $\left(S_{\mathrm{K}-16256, \text { ethanol }}=-0.20\right)$ with ethanol condensate, we assume only the wetting-ridge-induced oil drainage is present. Hence, the amount of oil drainage during ethanol condensation is $(60$ droplets $/ \mathrm{min}) \times\left(1.78 \times 10^{-14} \mathrm{~m}^{3}\right)=$ $1.07 \times 10^{-12} \mathrm{~m}^{3} / \mathrm{min}$.

Due to the positive spreading coefficient of K-16256 $\left(S_{\mathrm{K}-16256 \text {,steam }}=2.70\right)$ with steam condensate, extra oil will drain with shedding droplets due to cloak layer formation along with the presence of the oil-ridge. Hence, the amount of oil drainage during steam condensation is (30 droplets $/ \mathrm{min}) \times\left(1.78 \times 10^{-14} \mathrm{~m}^{3}+1.06 \times 10^{-13} \mathrm{~m}^{3}\right)=3.7 \times 10^{-12} \mathrm{~m}^{3} / \mathrm{min}$.

Furthermore, at the initiation of the experiments, SLIPS with $\mathrm{CuO}$ microstructures (structure height $h \approx 2 \mu \mathrm{m}$, solid fraction $\varphi \approx 0.023)$ have approximately $2 \mathrm{~mL} / \mathrm{m}^{2}\left(\approx 2 \times 10^{-6}\right.$ $\mathrm{m}^{3} / \mathrm{m}^{2}$ ) of lubricant per unit condenser surface area. ${ }^{8}$ So, the total amount of impregnated oil on a 3" long and 3/8" diameter SLIPS is $\pi \times 0.0095 \mathrm{~m} \times 0.0762 \mathrm{~m} \times 2 \times 10^{-6} \mathrm{~m}^{3} / \mathrm{m}^{2}=$ $4.55 \times 10^{-9} \mathrm{~m}^{3}$

Hence, the ideal lifespan ( $t_{\text {working fluid }}$ ) for a K-16256 infused SLIPS during ethanol and steam condensation are:

$t_{\text {ethanol }}=\left(4.55 \times 10^{-9} \mathrm{~m}^{3}\right) /\left(1.07 \times 10^{-12} \mathrm{~m}^{3} / \mathrm{min}\right)=4252.34 \mathrm{~min}=\mathbf{2 . 9 5}$ days.

$t_{\text {steam }}=\left(4.55 \times 10^{-9} \mathrm{~m}^{3}\right) /\left(3.7 \times 10^{-12} \mathrm{~m}^{3} / \mathrm{min}\right)=1229.73 \mathrm{~min}=\mathbf{0 . 8 5}$ days.

Similarly, the lifespan of a SLIPS infused with an order of magnitude lower viscosity oil $(\mathrm{K}-1525)$ is:

$t_{\text {ethanol, } \mathrm{K}-1525}=\left(4.55 \times 10^{-9} \mathrm{~m}^{3}\right) /\left(3.7 \times 10^{-11} \mathrm{~m}^{3} / \mathrm{min}\right)=122.97 \mathrm{~min}=\mathbf{2 . 0 5}$ hours, 
where 90 ethanol condensate droplets shed per minute maintaining a negative spreading coefficient $\left(S_{\mathrm{K}-1525, \text { ethanol }}=-0.20\right)$ with the infused oil. And the oil-ridge volume for K-1525 is, $V_{\text {ridge }}=$ $4.09 \times 10^{-13} \mathrm{~m}^{3}$.

The predicted lifespan of SLIPSs is far less than the actual timeline observed in the experiments (Figure 2 and Figure 3 of the manuscript). Although qualitatively the scaled lifetimes are in agreement (i.e. ethanol condensation results in lower SLIPS drainage and lower viscosity oil drains faster), multiple factors lead to the variation between the experimentally observed and theoretical estimated lifetime of the SLIPS. Although our scaling analysis is elucidating in identifying the volumetric contribution of lubricant loss due to cloaking and oil ridge formation, it does not consider kinetics. The cloak layer formation timescale or oil ridge formation timescale depend on the viscosity of the infused oils, the method of condensate formation (deposition vs condensation), as well as the shedding rate and droplet lifetime on the surface. Past work has shown that the initial cloak layer formation timescale for higher viscosity oils (K-16256) is an order of magnitude higher than moderate viscosity oils (K-1525, F-Y25/6). ${ }^{9}$ Also, the theoretical model assumed a uniform cloak layer thickness irrespective to the droplet size and oil viscosity. However, the cloaking layer is spatially and temporally non-uniform when compared to the lubricant underneath and behind a moving droplet. ${ }^{7}{ }^{10}$ Moreover, for simplification, we assumed same shedding droplet size, and similar droplet shedding velocity irrespective to the SLIPS surfaces and condensation environments (steam/ethanol). Oil depletion due to the oil ridge formation is a function of the departure velocity and oil viscosity. As the viscosity of the infused oil increases, the departure velocity decreases. ${ }^{10}$ Depending on the droplet size, not only will the shedding velocity change, but so will the cloak layer thickness and oil-ridge pattern. 
Moreover, the model used assumes a scenario of a single droplet moving on a SLIPS surface at a relatively constant velocity. This condition differs from that of droplet being formed, coalescing, and shedding during long-term condensation experiments (as conducted in this study). The droplet dynamics studied here involve multi-droplet coalescence, departure, and re-nucleation. In reality, continuous droplet-nucleation-re-nucleation, multi-droplet coalescence, and restoring/wicking phenomena exist ${ }^{11}$, which together lead to the continuous formation-rupturereformation of the cloaking layer and oil-ridge. The existing theoretical framework used for the scaling analysis and lifespan estimate do not capture these kinetics.

In addition, we assume that the total volume of oil present in the cloak layer and oil ridge drain with the shedding droplet and that all droplets shedding from a surface contribute equally to the oil depletion throughout the lifespan of the surface. In reality, these are simplified assumptions. As the infused oil starts to drain, it leads to the gradual exposure of the $\mathrm{CuO}$ microstructure tips, changing the interfacial phenomena between the condensate and underlying oil-infused microstructures. We hypothesize that overtime, due to this reduction of oil volume fraction, the timescale of the cloak layer and oil-ridge formation increases, which decreases the oil drainage rate and results in enhanced lifespan of the surfaces as observed in our experiments.

Future studies are needed to validate our hypotheses and to develop higher fidelity models of SLIPS lifespan that focus on the effects of oil viscosity, type of working fluid, and multi-droplet coalesce dynamics on cloaking oil layer formation, oil ridge formation, droplet shear dynamics, and time dependent oil-drainage, which all holistically combine to govern lubricant depletion dynamics on SLIPS. 


\section{S5. SEM Images}

a

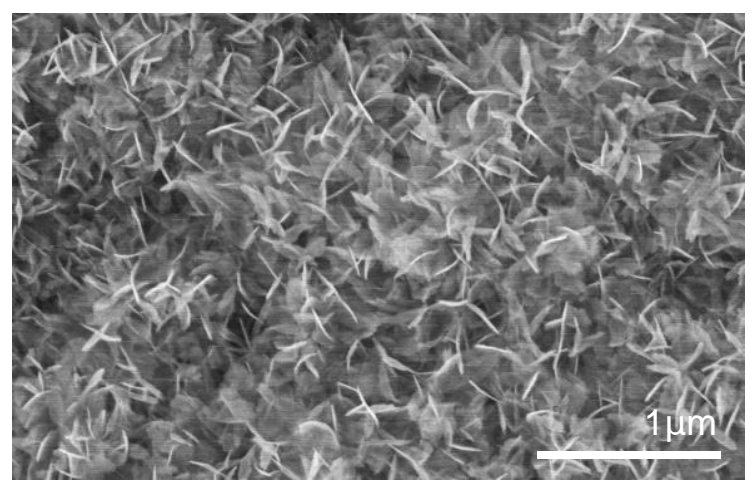

b

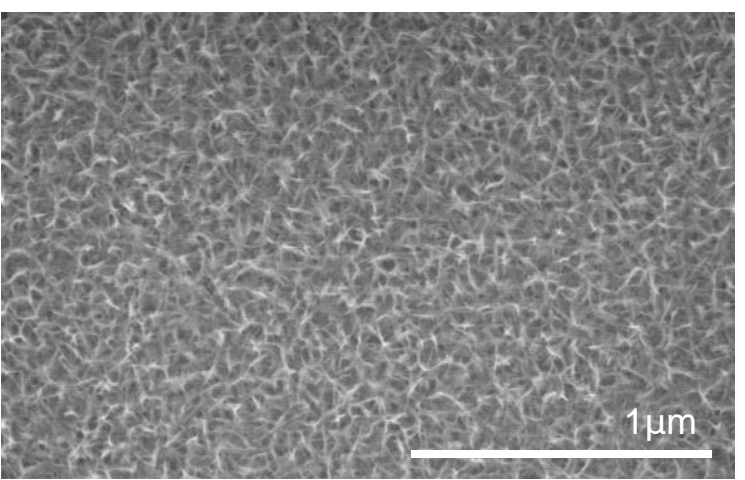

Figure S1. Scanning electron microscopy (SEM) images of boehmite nanostructures on the (a) Al tube and a (b) aluminum sputtered silicon wafer surface. 


\section{S6. Re-lubricated SLIPS Samples}

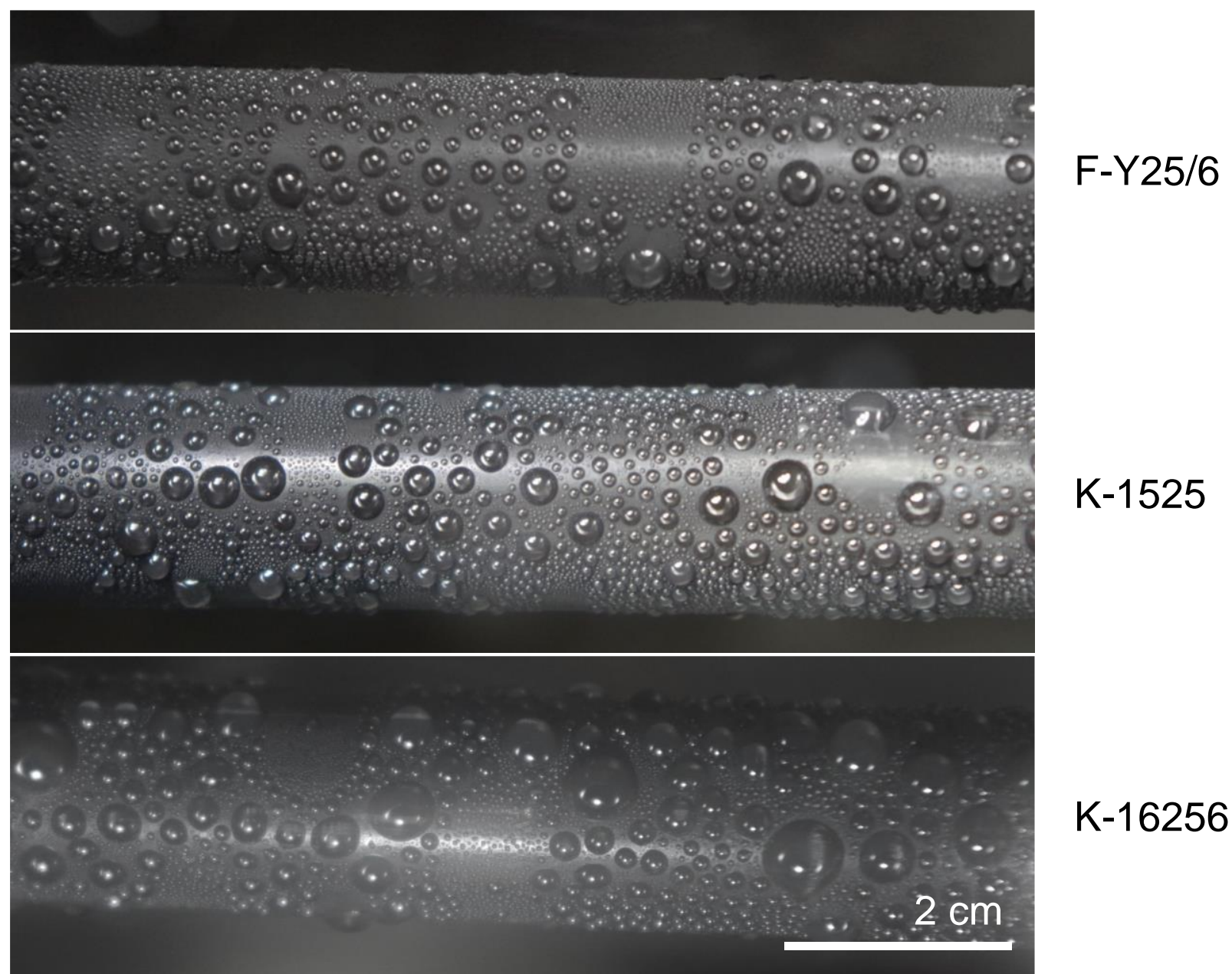

Figure S2. Ethanol condensation on re-lubricated SLIPSs after log-term durability test. After contiouns durability test the failed SLIPS samples were re-lubricated with fresh oil and condensation experiment was conducted in the same chamber. Re-lubricated samples show similar condensation behavior as of fresh samples. 


\section{S7. Effect of Oil Viscosity to the Heat Transfer Coefficient}

The overall heat transfer performance from the vapor to the cooling water can be expressed as a series sum of four individual thermal resistances: the internal convective resistance $R_{\text {conv }}$, the conduction copper tube wall resistance $R_{\text {wall }}$, the conductive resistance imposed by the nanostructures and infused lubricant oil $R_{\text {coating, }}$, and the condensation heat transfer resistance $R_{\text {condensation }}$.

Considering the maximum coating resistance, i.e. $t=2 \mu \mathrm{m}$ layer $(\approx$ height of $\mathrm{CuO}$ nanostructures) of lubricant on the tube outer surface given $k_{\text {Krytox }} \ll k_{\text {CuO }}\left(k_{\text {Krytox }} \sim 0.25 \mathrm{~W} /\right.$ $\left.(\mathrm{m} \cdot \mathrm{K}), k_{\mathrm{CuO}} \sim 33 \mathrm{~W} /(\mathrm{m} \cdot \mathrm{K})\right)$, the thermal resistance of the coating for a $\mathrm{Cu}$ tube with outer diameters, $d_{\mathrm{OD}}=9.52 \mathrm{~mm}$, inner diameters, $d_{\mathrm{ID}}=8.73 \mathrm{~mm}$, and lengths $L=108 \mathrm{~mm}$ is:

$$
R_{\text {coating }}=\frac{\ln \left(\frac{d_{\mathrm{OD}}+t}{d_{\mathrm{OD}}}\right)}{2 \pi L k_{\text {Krytox }}} \approx 1.23 \times 10^{-3} \frac{\mathrm{K}}{\mathrm{W}} .
$$

Similarly, the conductive tube wall resistance can be calculated by knowing the thermal conductivity of the wall $\left(k_{\mathrm{Cu}}=386 \mathrm{~W} /(\mathrm{m} \cdot \mathrm{K})\right.$, and using:

$$
R_{\mathrm{wall}}=\frac{\ln \left(\frac{d_{\mathrm{OD}}}{d_{\mathrm{ID}}}\right)}{2 \pi L k_{\mathrm{Cu}}} \approx 3.3 \times 10^{-4} \frac{\mathrm{K}}{\mathrm{W}} .
$$

Compared to these two resistances, for a standard experimental conditions $\left(\mathrm{h}_{\mathrm{i}} \approx 25 \mathrm{~kW} /\left(\mathrm{m}^{2} \cdot \mathrm{K}\right)\right.$, $\left.\mathrm{h} \approx 10 \mathrm{~kW} /\left(\mathrm{m}^{2} \cdot \mathrm{K}\right)\right)$ the internal convective resistance and condensation heat transfer resistance are significantly larger., ${ }^{4}, 12$

$$
R_{\text {conv }}=\frac{1}{h_{\mathrm{i}} \pi d_{\mathrm{ID}} L} \approx 0.0135 \frac{\mathrm{K}}{\mathrm{W}},
$$




$$
R_{\text {condensation }}=\frac{1}{h \pi d_{\mathrm{OD}} L} \approx 0.03 \frac{\mathrm{K}}{\mathrm{W}} \text {, }
$$

Thus, both the tube wall and the additional coating thermal resistances are significantly lower than condensation and internal convection resistances.

Moreover, the thermal conductivity of the lubricant oils does not vary with oil viscosity. For all viscosities of the oil tested here, the thermal conductivity of the oils ranges from $0.1 \mathrm{~W} /(\mathrm{m}$. $\mathrm{K})$ to $0.25 \mathrm{~W} /(\mathrm{m} \cdot \mathrm{K})$, even though the viscosity ranges by orders of magnitude. 


\section{S8. Durability Chamber Operation}

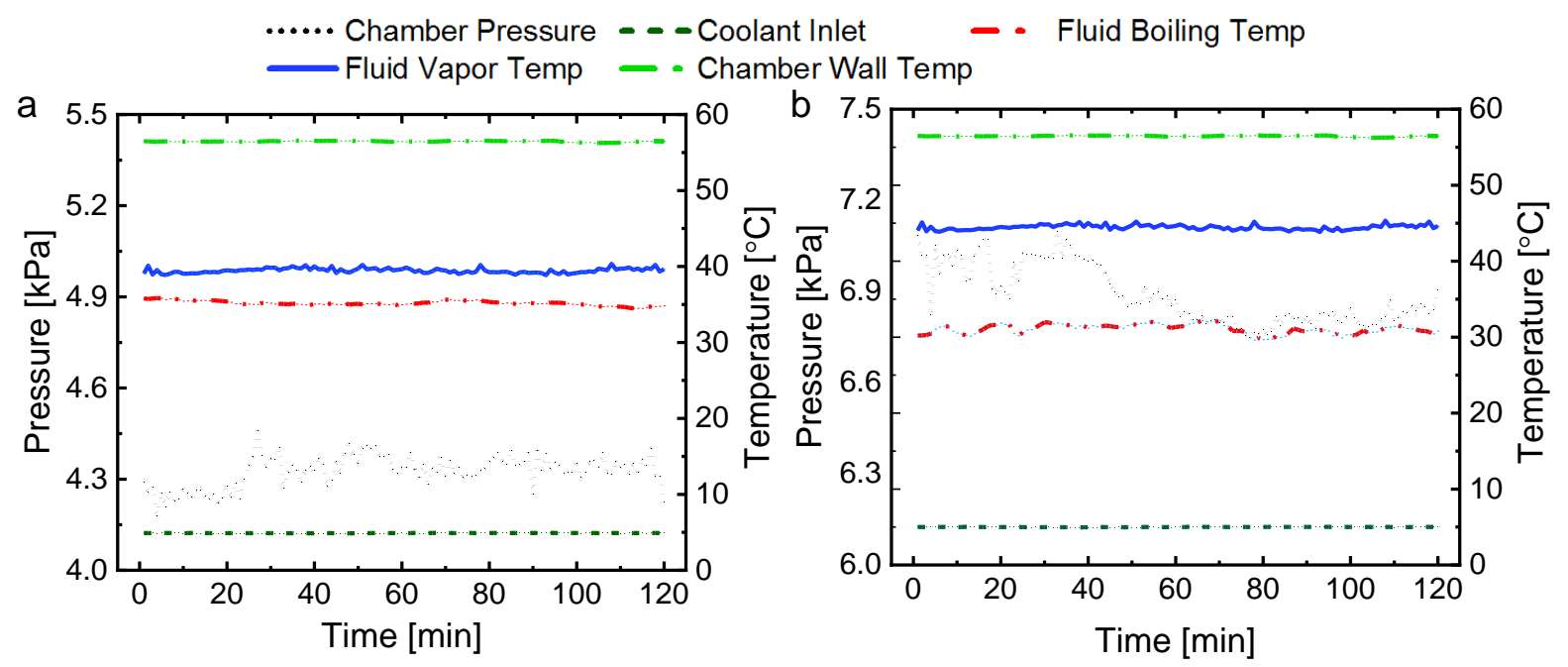

Figure S3. Steady state operating parameters of the durability chamber as a function of time with the working fluid (a)water, and (b) ethanol, respectively. Legends on the top are same for both (a) and (b). 


\section{References}

(1) Miljkovic, N.; Enright, R.; Nam, Y.; Lopez, K.; Dou, N.; Sack, J.; Wang, E. N. Jumpingdroplet-enhanced condensation on scalable superhydrophobic nanostructured surfaces. Nano letters 2012, 13 (1), 179-187.

(2) Enright, R.; Miljkovic, N.; Dou, N.; Nam, Y.; Wang, E. N. Condensation on superhydrophobic copper oxide nanostructures. Journal of Heat Transfer 2013, 135 (9), 091304. (3) Nam, Y.; Ju, Y. S. A comparative study of the morphology and wetting characteristics of micro/nanostructured $\mathrm{Cu}$ surfaces for phase change heat transfer applications. Journal of Adhesion Science and Technology 2013, 27 (20), 2163-2176.

(4) Sett, S.; Sokalski, P.; Boyina, K.; Li, L.; Rabbi, K. F.; Auby, H.; Foulkes, T.; Mahvi, A.; Barac, G.; Bolton, L. W.; Miljkovic, N. Stable Dropwise Condensation of Ethanol and Hexane on Rationally Designed Ultrascalable Nanostructured Lubricant-Infused Surfaces. Nano Lett 2019, 19 (8), 5287-5296, DOI: 10.1021/acs.nanolett.9b01754.

(5) Zhou Yang, Y.-Z. W., Yi-Fan Ye, Mao-Gang Gong and Xiao-Liang Xu. A Simple Way to Fabricate an Aluminum Sheet with Superhydrophobic and Self-Cleaning Properties. Chinese Physics B 2012, 21 (12), 126801.

(6) Miljkovic, N.; Enright, R.; Wang, E. N. Modeling and Optimization of Superhydrophobic Condensation. Journal of Heat Transfer 2013, 135 (11).

(7) Kreder, M. J.; Daniel, D.; Tetreault, A.; Cao, Z. L.; Lemaire, B.; Timonen, J. V. I.; Aizenberg, J. Film Dynamics and Lubricant Depletion by Droplets Moving on Lubricated Surfaces. Physical Review X 2018, 8 (3), 031053, DOI: ARTN 031053

10.1103/PhysRevX.8.031053.

(8) Sett, S.; Oh, J.; Cha, H.; Veriotti, T.; Bruno, A.; Yan, X.; Barac, G.; Bolton, L. W.; Miljkovic, N. Lubricant-Infused Surfaces for Low-Surface-Tension Fluids: The Extent of Lubricant Miscibility. ACS Appl Mater Interfaces 2021, 13 (19), 23121-23133, DOI:

10.1021/acsami.1c02716.

(9) Günay, A. A.; Sett, S.; Ge, Q.; Zhang, T.; Miljkovic, N. Cloaking Dynamics on LubricantInfused Surfaces. Advanced Materials Interfaces 2020, 7 (19), 2000983, DOI:

10.1002/admi.202000983.

(10) Adera, S.; Alvarenga, J.; Shneidman, A. V.; Zhang, C. T.; Davitt, A.; Aizenberg, J. Depletion of Lubricant from Nanostructured Oil-Infused Surfaces by Pendant Condensate Droplets. ACS Nano 2020, 14 (7), 8024-8035, DOI: 10.1021/acsnano.9b10184.

(11) Junyoung Lee, S. S., and Nenad Miljkovic, Opto-Hydrodynamic Characterization of Lubricant Infused Surfaces Degradation. 2022.

(12) Sett, S.; Sokalski, P.; Mehta, M.; Rabbi, K. F.; Gunay, A.; Miljkovic, N. Transient pulse condensation. Applied Physics Letters 2020, 117 (9), 091602, DOI: Artn 091602

$10.1063 / 5.0015311$. 